\title{
Superior PSZ-SOD Gap-Fill Process Integration Using Ultra-Low Dispensation Amount in STI for 28 nm NAND Flash Memory and Beyond
}

\author{
Chun Chi Lai, Yi Wen Lu, Hung Ju Chien, and Tzung Hua Ying \\ Powerchip Technology Corporation, No. 12, Li-Hsin 1st Road, Hsinchu Science Park, Hsinchu 30078, Taiwan \\ Correspondence should be addressed to Chun Chi Lai; agii@powerchip.com
}

Received 11 September 2014; Revised 8 April 2015; Accepted 15 April 2015

Academic Editor: Abdelwahab Omri

Copyright (C) 2015 Chun Chi Lai et al. This is an open access article distributed under the Creative Commons Attribution License, which permits unrestricted use, distribution, and reproduction in any medium, provided the original work is properly cited.

\begin{abstract}
The gap-fill performance and process of perhydropolysilazane-based inorganic spin-on dielectric (PSZ-SOD) film in shallow trench isolation (STI) with the ultra-low dispensation amount of PSZ-SOD solution have been investigated in this study. A PSZ-SOD film process includes liner deposition, PSZ-SOD coating, and furnace curing. For liner deposition, hydrophilic property is required to improve the contact angle and gap-fill capability of PSZ-SOD coating. Prior to PSZ-SOD coating, the additional treatment on liner surface is beneficial for the fluidity of PSZ-SOD solution. The superior film thickness uniformity and gap-fill performance of PSZ-SOD film are achieved due to the improved fluidity of PSZ-SOD solution. Following that up, the low dispensation rate of PSZ-SOD solution leads to more PSZ-SOD filling in the trenches. After PSZ-SOD coating, high thermal curing process efficiently promotes PSZ-SOD film conversion into silicon oxide. Adequate conversion from PSZ-SOD into silicon oxide further increases the etching resistance inside the trenches. Integrating the above sequence of optimized factors, void-free gap-fill and well-controlled STI recess uniformity are achieved even when the PSZ-SOD solution dispensation volume is reduced 3 to 6 times compared with conventional condition for the $28 \mathrm{~nm}$ node NAND flash and beyond.
\end{abstract}

\section{Introduction}

Several methods such as high density plasma and thermal chemical vapor deposition have been widely used in shallow trench isolation (STI) for NAND flash memory. As the memory device is scaled down to the $28 \mathrm{~nm}$ node and beyond, these methods exhibit poor gap-fill performance [1]. To overcome this problem, perhydropolysilazane-based inorganic spin-on dielectric (PSZ-SOD) film has been adopted due to its excellent gap-fill ability. In addition, PSZ-SOD film owns the quality of low moisture and high etching resistance [24]. However, the PSZ-SOD chemical is expensive, and waste liquid generated after the PSZ-SOD process would produce hydrogen gas, a highly explosive material. For reducing the cost and risk of waste liquid handling issue, it is important to decrease the dispensation amount of PSZ-SOD solution on wafer. Although using ultra-low amount of PSZ-SOD solution can avoid the above disadvantages, it would reduce the fluidity of PSZ-SOD solution. The reduction of PSZ-SOD solution fluidity is detrimental for gap-fill capability and conversion ratio after furnace curing of PSZ-SOD film. Furthermore, the recess uniformity in the trenches is hard to control in following STI etching back processes. Therefore, the $V$ th shifts and the reliability of device degrades [58]. For the breakthrough of the above-mentioned challenge, this paper presents several unique processes to improve the gap-fill capability of ultra-low amount PSZ-SOD solution by optimizing the factors including the film adsorption, fluidity, dispensation rate, and conversion ratio. Eventually, wellcontrolled recess uniformity is obtained.

\section{Experimental}

Figure 1(a) shows the process flow of PSZ-SOD in STI for the $28 \mathrm{~nm}$ node NAND flash. In the very beginning, conventional and hydrophilic liners were prepared for comparison. The hydrophilicity of liner surface was defined by contact angle. The conventional dispensation amount of PSZ-SOD solution was $2 \sim 4 \mathrm{cc}$. for PSZ-SOD coating. The extra surface treatment 


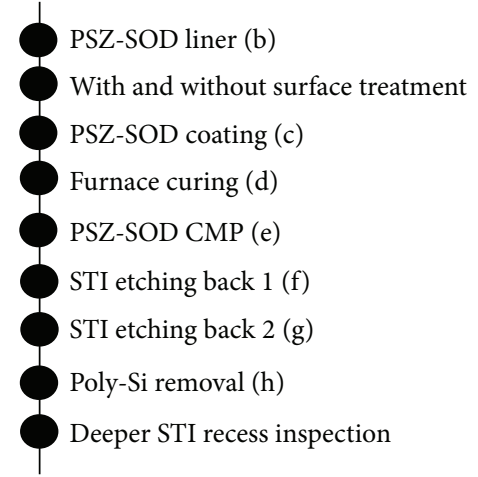

(a)

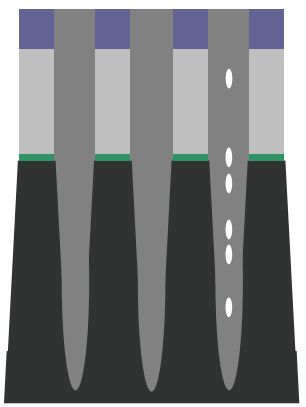

(e)

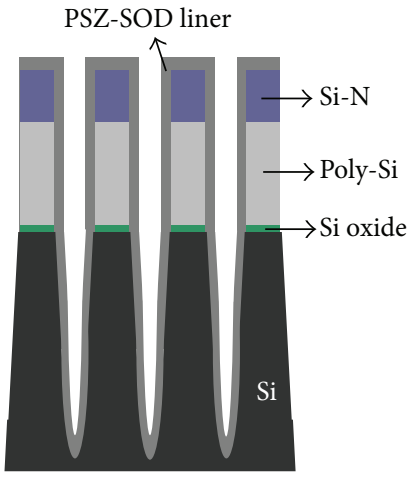

(b)

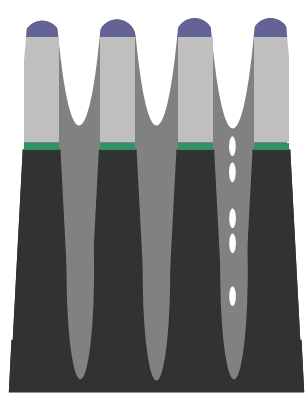

(f)

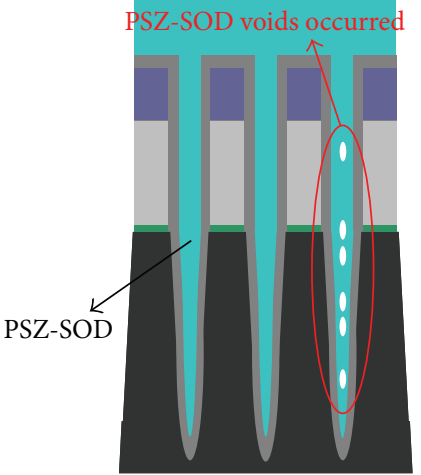

(c)

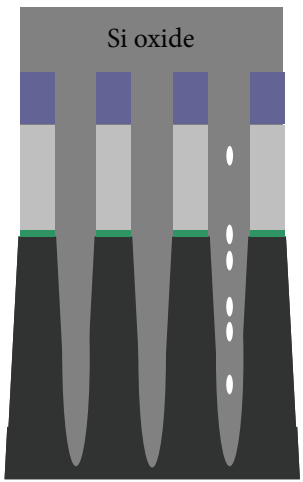

(d)

PSZ-SOD voids induce Poly-Si is removed to reveal deeper STI recess deeper STI recess

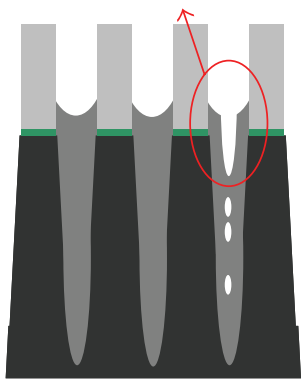

(g)

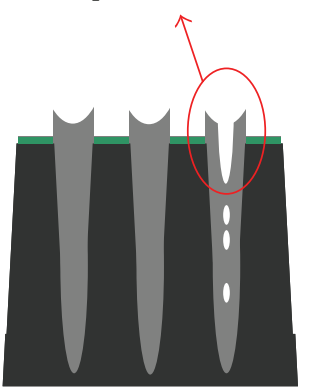

(h)

FIgure 1: (a) The flow of PSZ-SOD process in STI for $28 \mathrm{~nm}$ node NAND flash. The schematics of PSZ-SOD flow: (b) PSZ-SOD liner deposition, (c) PSZ-SOD coating, (d) furnace curing for PSZ-SOD converting to silicon oxide, (e) chemical-mechanical polishing of silicon oxide, (f) STI etching back 1 process, (g) STI etching back 2 process, and (h) removing of polysilicon of floating gate.

step was inserted between liner deposition and PSZ-SOD coating. The purpose of the extra surface treatment is the enhancement of surface hydrophilicity. The amount of PSZSOD solution, as low as $0.6 \sim 2$ cc., was dispensed for realizing the feasibility of the hydrophilic-enhanced surface. $600 \mathrm{~nm}$ thick PSZ-SOD films with varied dispensation amount and rate were subsequently coated on the wafer. Following that up, the furnace curing process was preceded at $280-500^{\circ} \mathrm{C}$ in the steam condition and $900^{\circ} \mathrm{C}$ in $\mathrm{N}_{2}$ ambient for converting the PSZ-SOD film into the silicon oxide. After chemicalmechanical polishing of silicon oxide and STI etching back process, the voids in STI induce the deeper STI recesses. Figures $1(\mathrm{~b}) \sim 1(\mathrm{~g})$ show the schematic representation of the above process flow. To reveal and inspect the recesses buried in STI, the polysilicon of floating gate was removed as shown in Figure 1(h). The deeper STI recess inspection by top view and cross-sectional scanning electron microscopy (SEM) image is presented in Figure 2. The counts of deeper STI recess determine the level of PSZ-SOD voids.

\section{Results and Discussion}

In order to realize the relationship between fluidity and solution dispensation amount of PSZ-SOD process, the PSZSOD solution was dispensed on blanket wafer first. Figure 3 is the results of the primary test on blanket wafers. As shown in Figure 3, the thickness uniformity (TU) of $600 \mathrm{~nm}$ thick SOD film varies with the dispensation amount of PSZSOD solution in comparison with low dispensation and conventional condition. Generally, the dispensation range, 2 4 cc., is used for conventional PSZ-SOD process. The TU is less than $3 \%$. The TU drastically increases to $25 \%$ as dispensation amount is less than $1.5 \mathrm{cc}$. The worse thickness uniformity results from the insufficient fluidity of PSZ-SOD solution in low dispensation condition. The worse fluidity also causes the discoloration of PSZ-SOD film as shown in Figure 4. The discoloring regions mean thickness deviation. During the furnace curing process of PSZ-SOD film, the nonuniformity thickness would induce the nonuniformity conversion ratio of silicon oxide to cause the worse wet etch resistance performance. The same result of discoloring performance was also confirmed on a pattern structure wafer without surface treatment. For the structure wafer coated by PSZ-SOD film without surface treatment, the discoloring film disappeared after increasing PSZ-SOD dispensation amount to $1.8 \mathrm{cc}$. It indicates that PSZ-SOD solution movement on the surface of structure wafer is stricter than that of blanket wafer because of patterning effect. However, by applying additional surface treatment on liner, the thickness uniformity of SOD film keeps lower than 3\% even with dispensation amount as low as $0.6 \mathrm{cc}$. The identical result is also observed on a pattern structure wafer. Thus, the surface treatment step provides 


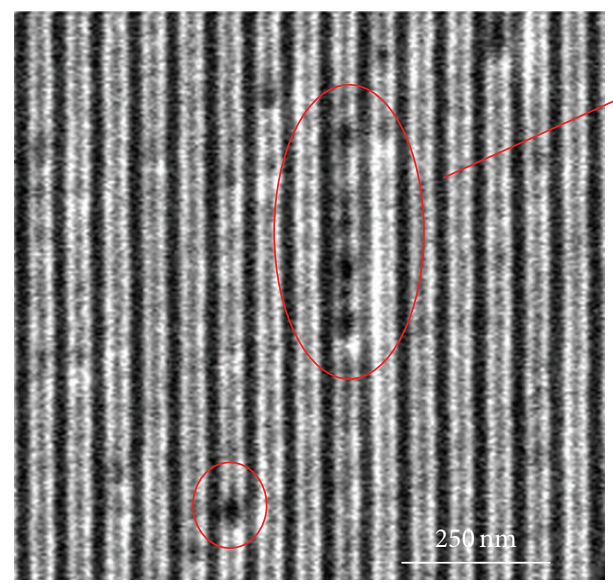

(a)

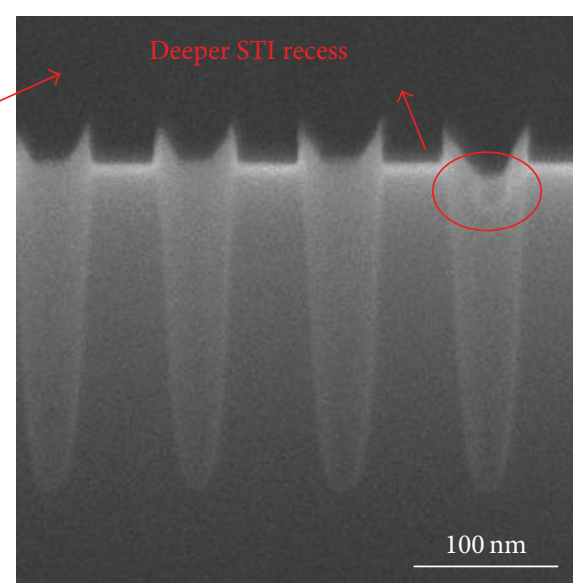

(b)

FIGURE 2: The deeper STI recess inspection by (a) top view and (b) cross-sectional SEM image.

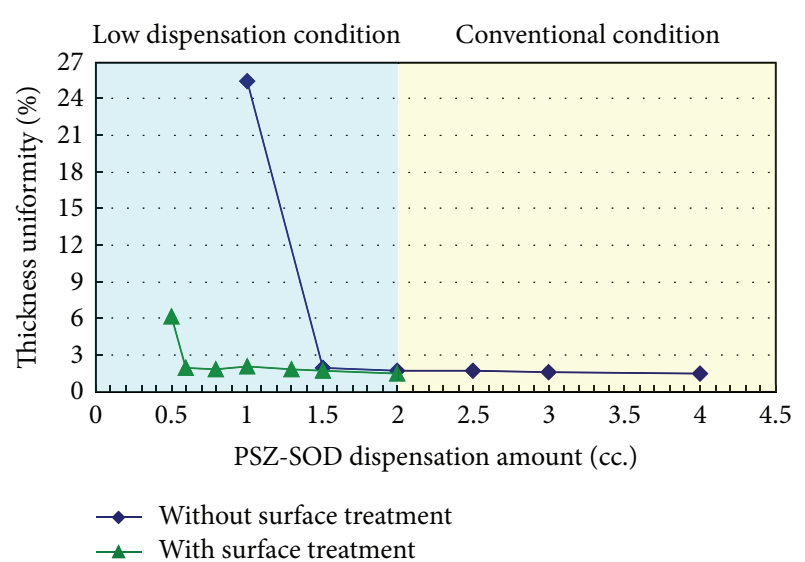

FIGURE 3: The thickness uniformity of blanket wafer by varied dispensation amount of PSZ-SOD solution with and without surface treatment step in low dispensation and conventional condition.

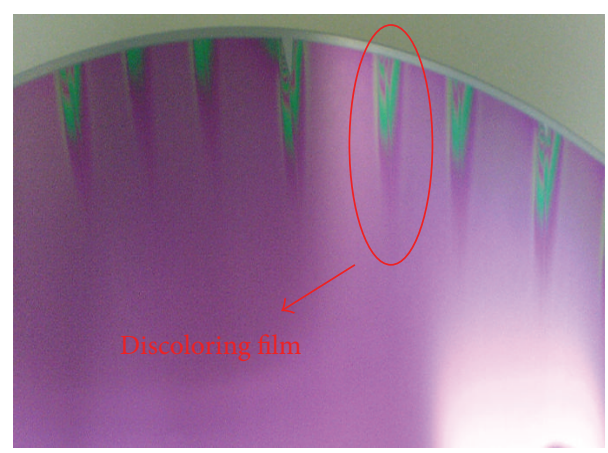

FIGURE 4: Discoloration of PSZ-SOD film in blanket wafer with less than $1.5 \mathrm{cc}$. dispensation amount of PSZ-SOD solution.

equivalent fluidity for both blanket and pattern structure wafers. The patterning effect is successfully eliminated by the surface treatment. Comparing with conventional PSZSOD process, 3 to 6 times reduction of PSZ-SOD solution

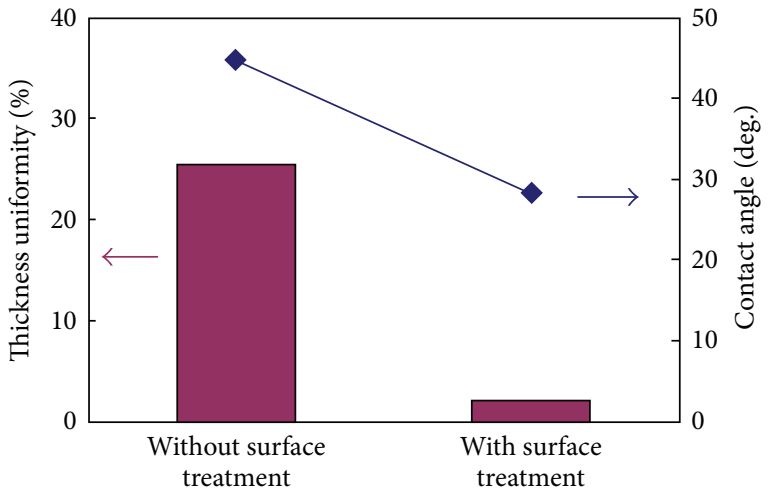

FIGURE 5: The relationship between surface contact angle and thickness uniformity with and without surface treatment step.

dispensation volume is achieved. The application of surface treatment step certainly improves the fluidity in the ultralow amount of PSZ-SOD $(0.6 \mathrm{cc}$.) solution especially for the structure wafer. Figure 5 shows the relationship between surface contact angle and TU with and without surface treatment step. The lower contact angle appears after surface treatment. Apparently, the surface treatment step enhances the hydrophilic property of PSZ-SOD liner to improve the fluidity of PSZ-SOD solution. Accordingly, the thickness uniformity of PSZ-SOD film strongly depends on the fluidity of PSZ-SOD solution. The required amount of PSZ-SOD solution dispensation is therefore decreased by increasing the fluidity of the solution as shown in Figure 3.

Regarding the film quality in ultra-low amount of PSZSOD solution with surface treatment step, the FTIR spectra and SIMS profiles are shown in Figure 6. About FTIR spectra of PSZ-SOD film, the same absorbance curves of functional groups between ultra-low amount of PSZ-SOD solution with surface treatment step and conventional condition are verified. After furnace curing of PSZ-SOD film, 


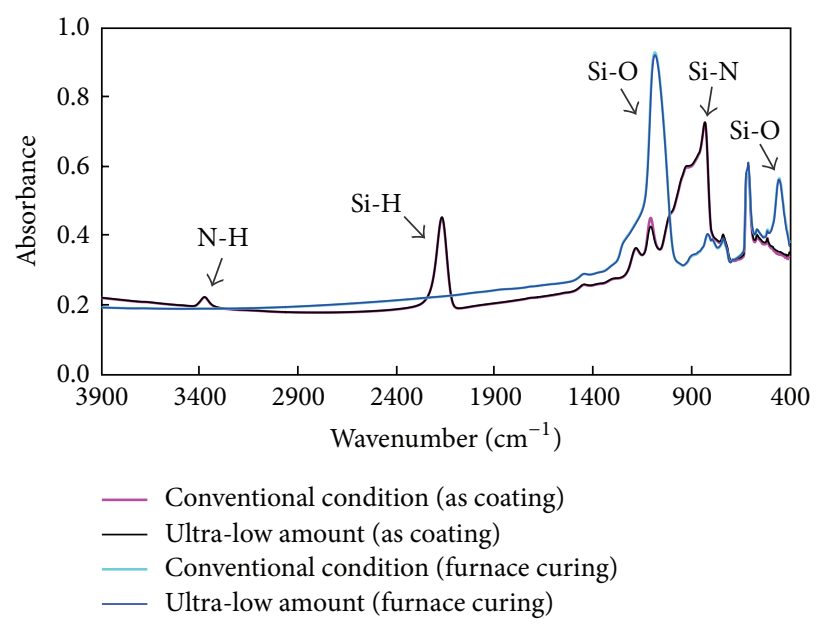

(a)

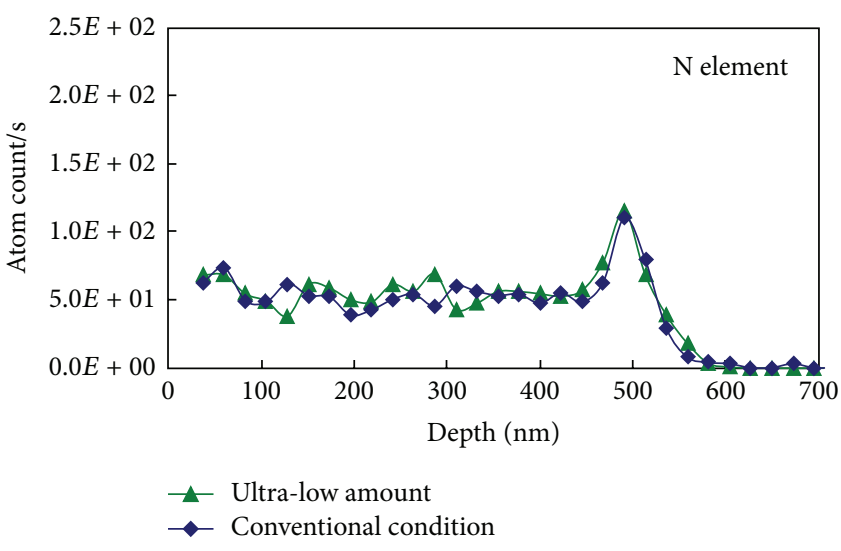

(b)

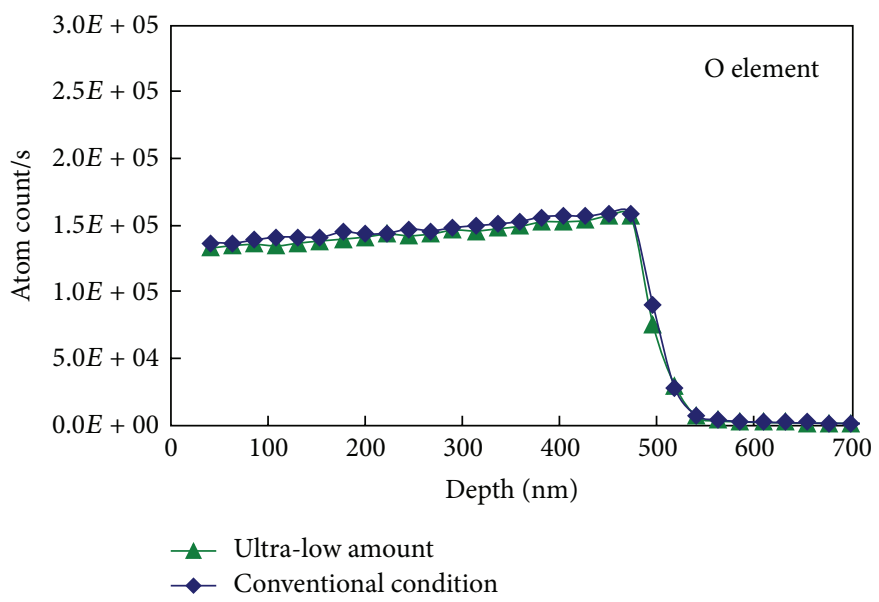

(c)

Figure 6: (a) The FTIR spectrum. SIMS depth profiles of (b) N and (c) O elements in ultra-low amount of PSZ-SOD solution with surface treatment step and conventional condition.

the absorbance of Si-H $\left(2100-2250 \mathrm{~cm}^{-1}\right)$ and N-H (3320$3420 \mathrm{~cm}^{-1}$ ) disappears. Furthermore, the performances of conversion from $\mathrm{Si}-\mathrm{N}$ bond $\left(825-875 \mathrm{~cm}^{-1}\right)$ to $\mathrm{Si}-\mathrm{O}$ bond $\left(440-460 \mathrm{~cm}^{-1}\right.$ and $\left.1057-1087 \mathrm{~cm}^{-1}\right)$ are the same in both conditions. From the SIMS analyses, the atom counts of nitrogen and oxygen present a similar tendency after furnace curing. Thus, the film composition from ultra-low amount of PSZ-SOD solution with surface treatment step is certainly identical with conventional condition.

Although the TU and the phenomena of discoloration with ultra-low amount of PSZ-SOD solution are improved by surface treatment step, the voids occur in the trenches as marked by circle in Figure 7(a). After etch back process, the existing voids become the deeper STI recesses as shown in Figure 7(b). The existence of voids implies that the horizontal movement of fluid is accomplished but vertical movement of fluid in the trenches is insufficient. For the enhancement of the gap-fill ability, the liner property was investigated. Figure 8 shows the counts of deeper STI recesses and contact angles for conventional and hydrophilic liners.
The conventional liner with more hydrophobic property generates less hydrogen bonding with the functional group of Si-NH in PSZ-SOD film. Consequently, the larger contact angle of conventional liner increases the number of deeper STI recesses. In contrast, the hydrophilic character strengthens the adsorption between PSZ-SOD film and liner. For the practical application, the contact angle and deeper STI recesses are significantly improved by the utility of hydrophilic liner.

About the surface treatment process, the relationship between deeper STI recesses in the trenches and the surface treatment time is displayed in Figure 9. Figure 9 shows deeper STI recess counts and surface contact angle as the function of surface treatment time. The long surface treatment time gets the low contact angle. Obviously, long surface treatment time can reduce the amount of deeper STI recess because the fluidity of PSZ-SOD solution is improved. Both the vertical and the horizontal movements of ultra-low amount of PSZ-SOD solution are reinforced by the elongation of surface treatment time. 


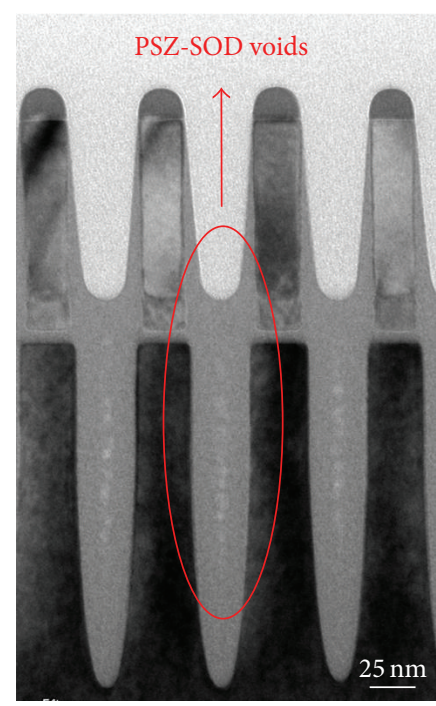

(a)

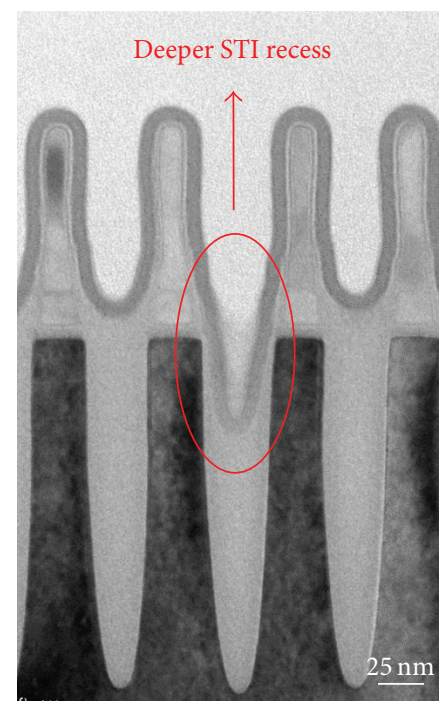

(b)

Figure 7: Cross-sectional transmission electron microscope (TEM) images after (a) etch back 1 process and (b) etch back 2 process.

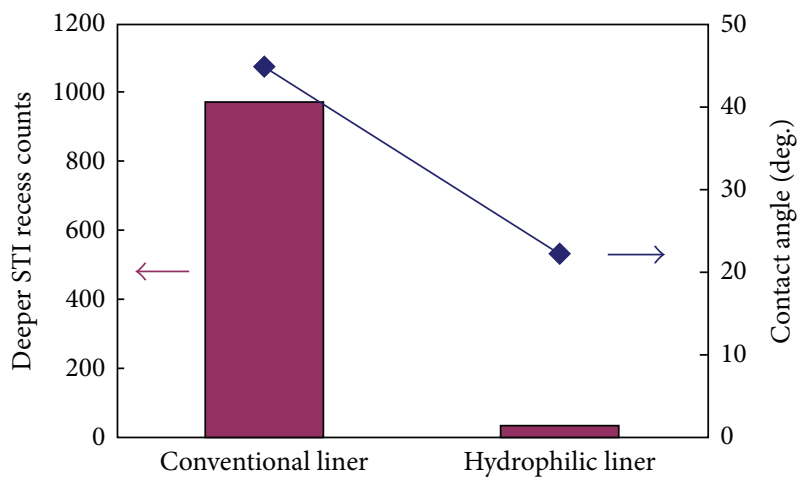

FIGURE 8: The relationship between deeper STI recess counts and contact angle with conventional and hydrophilic liners.

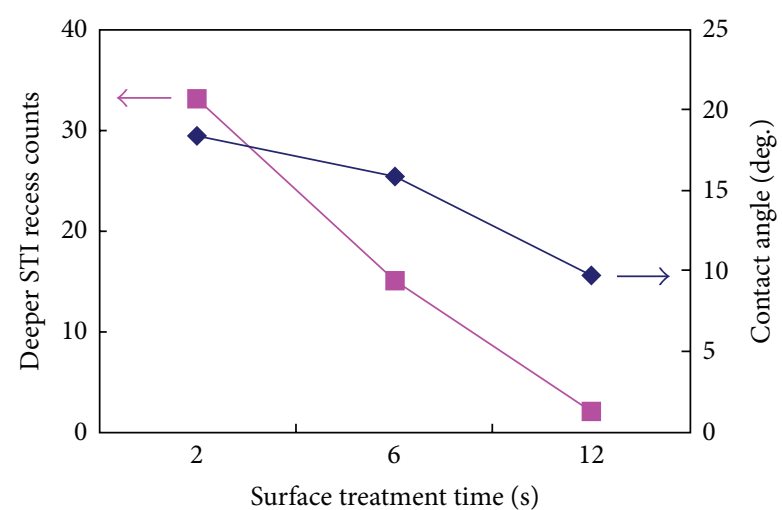

FIGURE 9: The deeper STI recess counts and surface contact angle performances with varied surface treatment time.

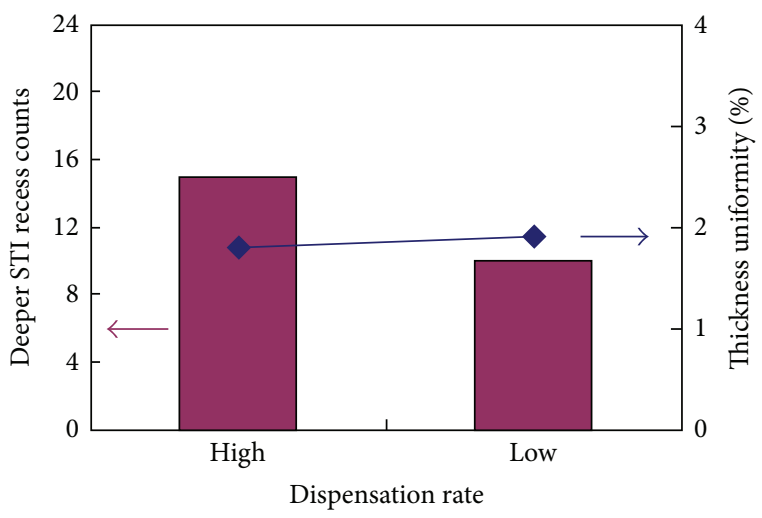

FIGURE 10: The result of TU and deeper STI recess counts with changing dispensation rate of PSZ-SOD solution.

The impact between dispensation rate of PSZ-SOD solution on the wafer and gap-fill ability is included in this research. Low dispensation rate extends the staying duration of PSZ-SOD solution on the wafer before spin-drying. As expected, Figure 10 indicates that lower dispensation rate decreases the number of deeper STI recess counts. The decrement of deeper STI recess counts is the consequence of gap-fill improvement. For the thickness uniformity of SOD film, the results of low and high dispensation rates are comparable.

According to above unique process refinements, the fine gap-fill is accomplished with ultra-low amount of PSZSOD solution as shown in Figure 11. However, after etching back process, few nonuniform recesses are discovered. Since the trench gap-fill ability of PSZ-SOD film with ultralow amount of PSZ-SOD solution is sufficient by fluidity 


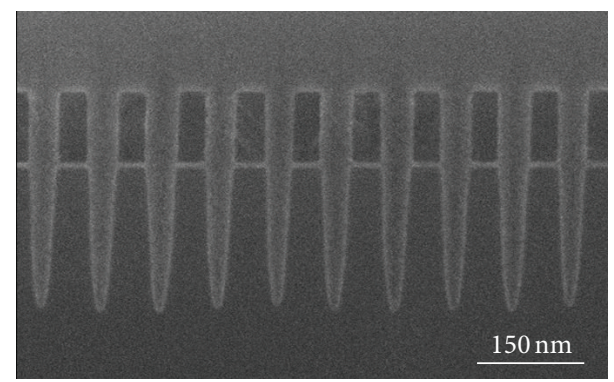

FIGURE 11: The SEM image of PSZ-SOD film coating with optimized PSZ-SOD process.

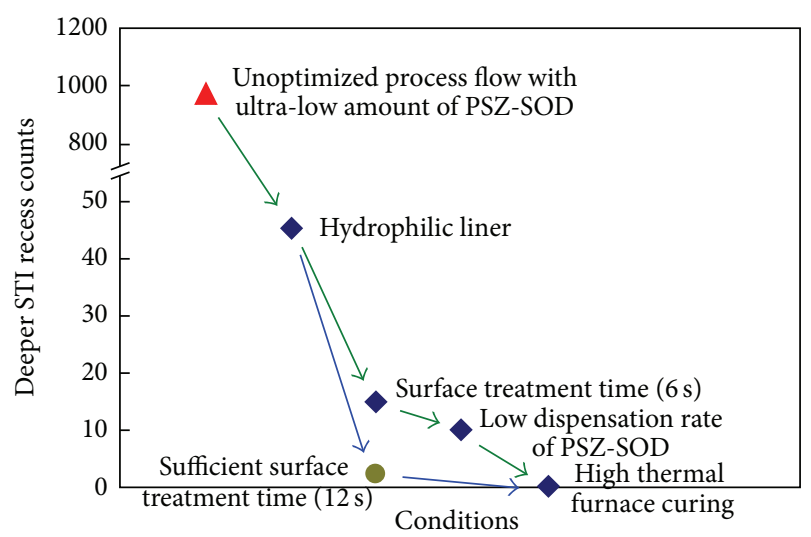

FIGURE 12: The deeper STI recess improvement status with optimized processes.

improvement, the nonuniform recesses are attributed to the poor conversion efficiency of PSZ-SOD during furnace curing process. The poor conversion efficiency makes PSZSOD film quality inconsistent in trenches. Subsequently, the deeper STI recesses appear during trench etching back process because the trenches are filled with the worse etching resistance PSZ-SOD film. Hence, the optimization of furnace curing temperature is necessary for enhancing the conversion efficiency of PSZ-SOD film. Figure 12 shows the deeper STI recess counts of optimized process flows including thermaldependent furnace curing conditions. Two optimized process flows are presented for the phenomenon of deeper STI recess improvement. After hydrophilic liner deposition, low dispensation rate of PSZ-SOD can compensate the insufficient surface treatment time. In other words, sufficient surface treatment time can receive the void-free gap-fill performance. Both optimized process flows are followed by high thermal furnace curing to reduce the few deeper STI recesses caused by various PSZ-SOD film qualities in trenches. High thermal furnace curing reveals the superior ability of PSZ-SOD film conversion into silicon oxide in both optimized flows. Finally, comparing with unoptimized process flow with ultra-low amount of PSZ-SOD solution, the well-controlled recess uniformity after etching back process is presented by the above unique process (see Figure 12). Since the above unique processes can significantly improve the gap-fill performance and control the trench recesses in the strict condition of ultralow PSZ-SOD amount, these methods could be introduced to conventional amount of PSZ-SOD to strengthen the process ability.

\section{Conclusions}

For the improvement of gap-fill ability and uniform recess controlling, the optimized integration process has been achieved in step-by-step investigation. For PSZ-SOD liner selection, the hydrophilic property is necessary for creating more hydrogen bonds with PSZ-SOD film to improve gap-fill. Prior to PSZ-SOD film coating on the wafer, surface treatment step certainly improves both vertical and horizontal movements by the enhancement of surface hydrophilicity and sufficient surface treatment time. During PSZ-SOD solution dispensation on the wafer, low dispensation rate extends the time of PSZ-SOD filling in the trenches. Finally, high thermal furnace curing is used for reinforcing the PSZSOD film conversion into consistent composition of silicon oxide. The void-free gap-fill performance in STI with ultralow amount of PSZ-SOD and uniform recesses after etching back processes are accomplished by these unique process refinements.

\section{Conflict of Interests}

The authors declare that there is no conflict of interests regarding the publication of this paper.

\section{References}

[1] W. Z. Wong, J. J. Fan, J. D. Jiang et al., "The impact on device characteristics with STI formed by spin-on dielectric in high density NAND flash memory," in Proceedings of the International Symposium on VLSI Technology, Systems, and Applications (VLSI-TSA '09), pp. 9-10, April 2009.

[2] Y. Homma, T. Furusawa, H. Morishima, and H. Sato, "Low permittivity dielectrics and global planarization for quartermicron multilevel interconnections," Solid-State Electronics, vol. 41, no. 7, pp. 1005-1011, 1997.

[3] J. H. Heo, S. J. Hong, D. H. Ahn et al., "Void free and low stress shallow trench isolation technology using P-SOG for sub $0.1 \mu \mathrm{m}$ device," in Proceedings of the Symposium On VLSI Technology Digest of Technical Papers, pp. 132-133, 2002.

[4] L. Prager, A. Dierdorf, H. Liebe et al., "Conversion of perhydropolysilazane into a SiOx network triggered by vacuum ultraviolet irradiation: access to flexible, transparent barrier coatings," Chemistry - A European Journal, vol. 13, no. 30, pp. 8522-8529, 2007.

[5] Z.-S. Wang, Y.-J. Lee, R. Yang, Y.-C. Li, H.-H. Chen, and C. J. Lin, "A new recess method for SA-STI nand flash memory," IEEE Electron Device Letters, vol. 33, no. 6, pp. 896-898, 2012.

[6] K. Kim and J. Choi, "Future outlook of NAND flash technology for 40nm node and beyond," in Proceedings of the 21st IEEE NonVolatile Semiconductor Memory Workshop (NVSMW'06), pp. 911, Monterey, Calif, USA, February 2006.

[7] M. Park, C.-S. Lee, S.-H. Hur, K. Kim, and W.-S. Lee, “The effect of field oxide recess on cell VTH distribution of NAND flash cell 
arrays," IEEE Electron Device Letters, vol. 29, no. 9, pp. 10501052, 2008.

[8] J. D. Choi, S. S. Cho, Y. S. Yim et al., "Highly manufacturable 1 Gb NAND flash using $0.12 \mu \mathrm{m}$ process technology," in Proceedings of the IEEE International Electron Devices Meeting (IEDM '01), pp. 25-28, usa, December 2001. 

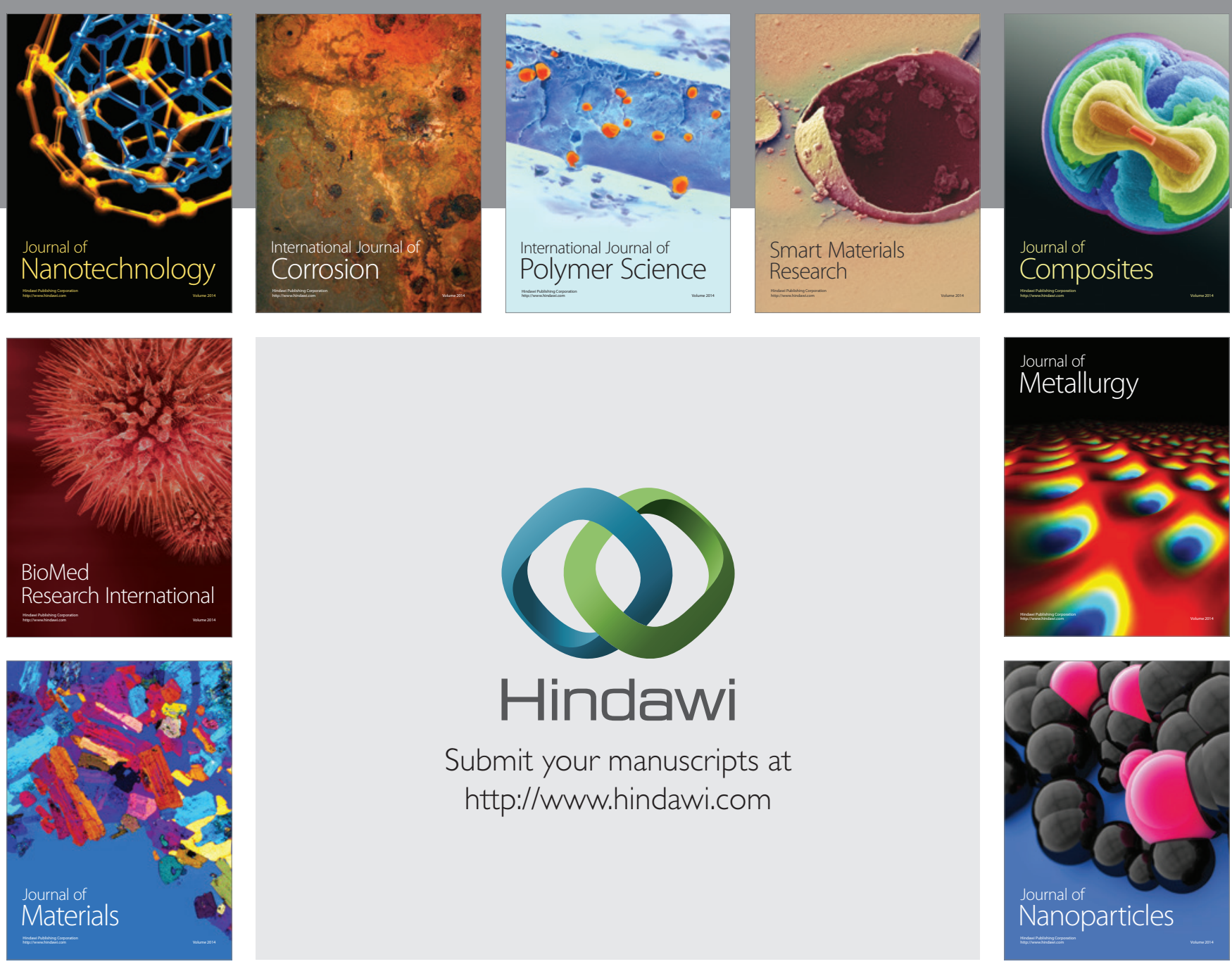

Submit your manuscripts at http://www.hindawi.com
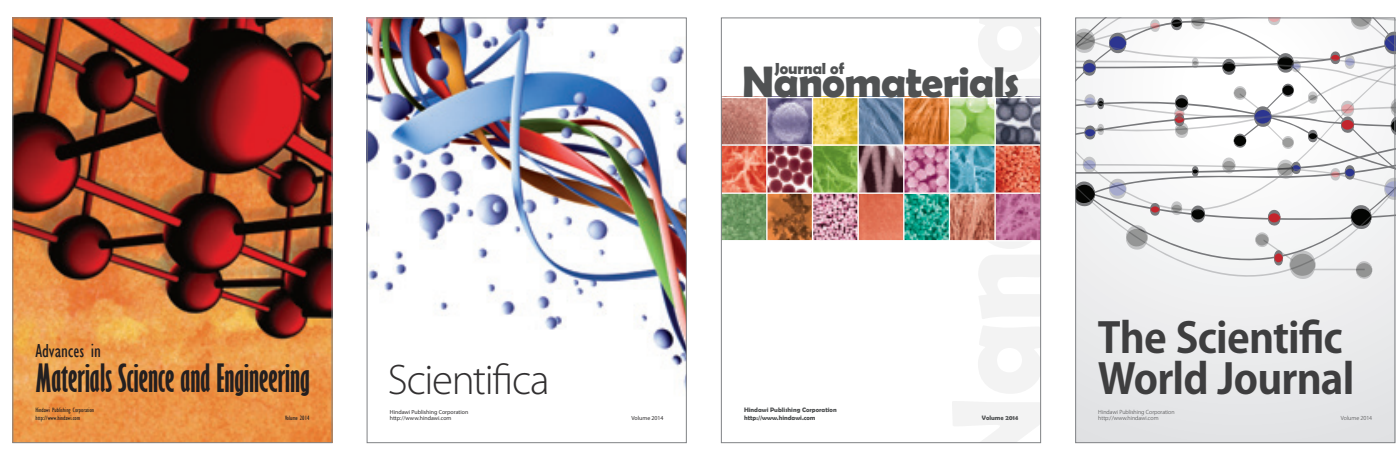

\section{The Scientific World Journal}
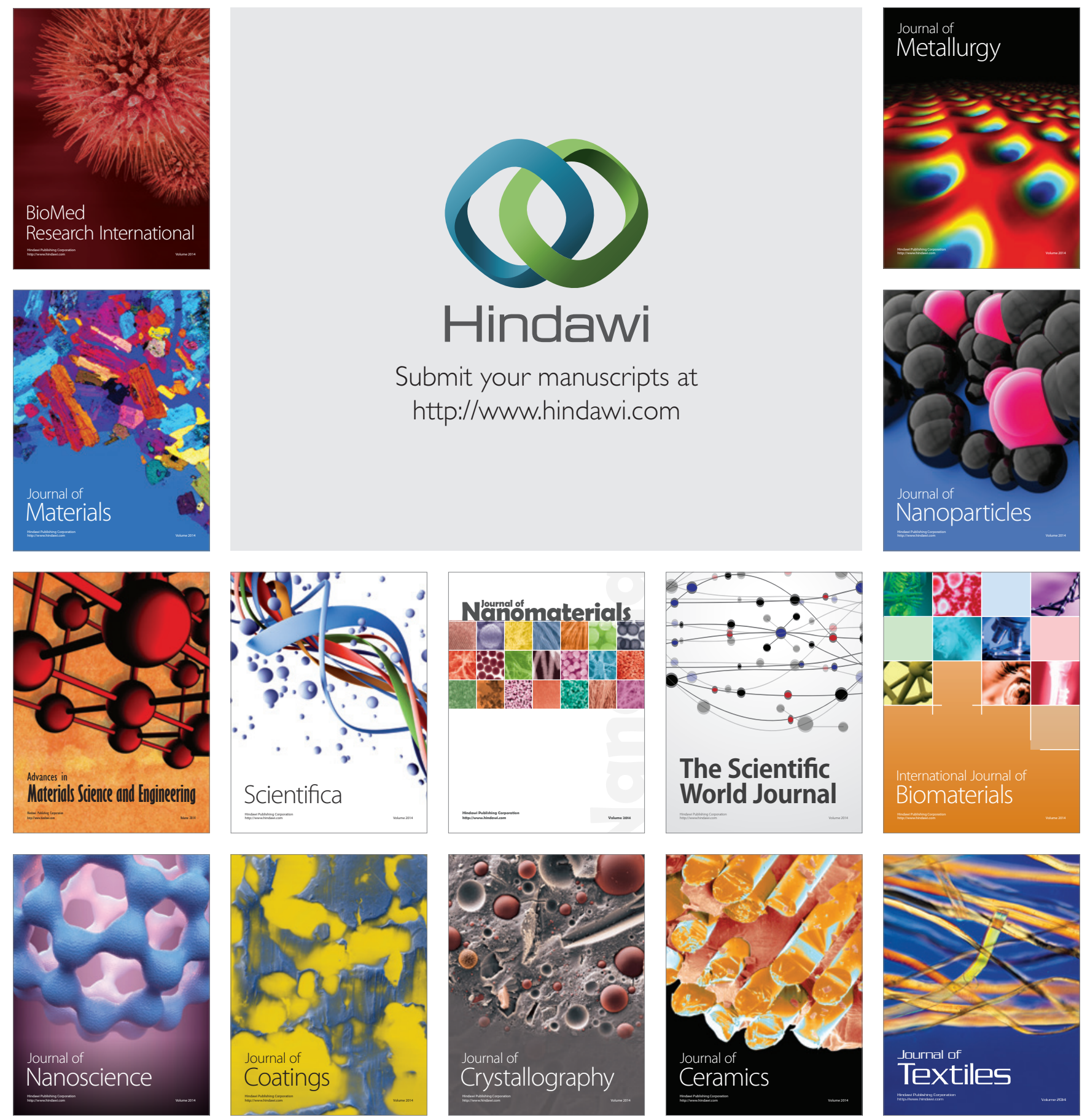